\title{
Near-infrared polarimetric and spectrometric study of the massive star-forming region $\mathrm{S} 87$
}

\author{
X. Chen ${ }^{1}$, Y. Yao ${ }^{2}$, J. Yang ${ }^{1,2}$, Z. Jiang ${ }^{1,2}$, and M. Ishii ${ }^{3}$ \\ 1 Purple Mountain Observatory, Chinese Academy of Sciences, 2 Beijing Westroad, Nanjing 210008, PR China \\ e-mail: xpchen@pmo.ac.cn \\ 2 National Astronomical Observatory, Chinese Academy of Sciences, Beijing 100012, PR China \\ e-mail: yqyao@bao.ac.cn; [jiyang;zbjiang]@pmo.ac.cn \\ 3 National Astronomical Observatory of Japan, 2-21-1 Osawa, Mitaka 181, Japan \\ e-mail: ishii@optik.mtk.nao.ac.jp
}

Received 10 May 2004 / Accepted 30 July 2004

\begin{abstract}
We present $J H K^{\prime}$ imaging polarimetry for the massive star-forming region $\mathrm{S} 87$, and $K$-band spectrometry from 2.0 to $2.35 \mu \mathrm{m}$ for several infrared sources in the region. The polarimetric patterns of S87E reveal a deeply embedded source (DES-S87) associated with reflection nebulosities. Exciting OH maser and $1.3 \mathrm{~mm}$ dust continuum emission, the DES-S87 is suggested to be the youngest star in S87 and the driving source of bipolar CO outflow in the region. A blue cavity is discovered in the $\left[J-K^{\prime}\right]$ color image, to the southwest of DES. Radial dependence of polarization degrees in the cavity could be fitted to the slab model of outflows at an inclination of $\sim 60^{\circ}$ out of the plane of the sky (Minchin et al. 1991b). The nebular complex of S87E can be divided into three area. The North nebula, exhibiting a bipolar structure, may represent a bipolar outflow in the northwest-southeast direction. The southwest nebula traces the blueshifted CO outflow and corresponds to the cavity structure discovered in the $\left[J-K^{\prime}\right]$ color image. The nebular structure of S87E suggests a quadrupolar outflow driven by DES-S87. A well-defined centrosymmetric pattern is found in the nebula of S87W with high degrees of polarization, which indicates single scattering off small dust grains in the reflection nebula. The polarimetric vectors around NIRS A show a polarization disk feature oriented in the southeast-northwest direction. Strong $\mathrm{Br} \gamma$ emission and $\mathrm{H}_{2}$ emissions are found in the $K$-band spectra, indicating the presences of strong stellar wind, envelope/disk, shocked gas, and high-density PDRs in the circumstellar environment of S87E and S87W. According to the $K$-band spectrum, the mass of DES-S87E is estimated to be $20 M_{\odot}$.
\end{abstract}

Key words. ISM: individual: objects S87 - ISM: lines and bands - polarization - stars: formation instrumentation: polarimeters

\section{Introduction}

S87 is well-known as a site of massive star formation at a distance of $2.1 \mathrm{kpc}$ (Clemens 1985). In the optical, S87 is a faint and diffuse nebulosity, extending over 10 arcmin. IRAS observations suggest it to be a strong far-infrared source at all observed wavelengths with the luminosity of $3.5 \times 10^{4} L_{\odot}$ (Barsony 1989). Near-infrared imaging presents two infrared reflection nebulae in the east and west of the region, denoted as S87E and S87W, respectively (Chen et al. 2003, hereafter Paper I). A young cluster was discovered within the nebula S87E (Paper I), where active star formation is indicated by an IRAS source, a compact HII region (Sharpless 1959), highly variable $\mathrm{H}_{2} \mathrm{O}$ masers (Blair et al. 1980; Henkel et al. 1986), and a massive bipolar outflow (Barsony 1989). On the other hand, the nebula S87W is relatively simple and evolved, in which two bright sources are obvious (Paper I).

VLA maps of the S87 HII region reveal a radio continuum source consisting of a compact core about $1^{\prime \prime}$ (0.6 pc for a distance of $2.1 \mathrm{kpc}$ ) in size and a fan-shaped tail approximately $7^{\prime \prime} \times 6$ " extending to the southeast (Bally \& Predmore 1983; Barsony 1989). Both Barsony and Bally \& Predmore concluded that a single B0 star could be responsible for the ionization of the HII region. CO observations show that the HII region coincides spatially with the peak $\mathrm{CO}$ emission from a 3.6 pc diameter molecular cloud (Bally 1981). A molecular cloud core, which is $\sim 2^{\prime \prime}$ in diameter and $500-1000 M_{\odot}$ in mass (Bally \& Lada 1983), is found in the center of the cloud. Traced by high spatial resolution $\mathrm{CS}(2-1)$ and ${ }^{13} \mathrm{CO}(1-0)$ lines, a northeast-southwest oriented molecular outflow has been discovered by Barsony (1989). The millimeter-wavelength data indicate that the bipolar outflow has a wide opening angle $\left(>60^{\circ}\right)$ and a substantial inclination angle $\left(\approx 50^{\circ}\right)$ (Barsony 1989).

Infrared imaging polarimetry serves as a powerful technique to study the morphology of star formation regions (e.g., Tamura et al. 1991). In infrared cluster regions, the polarization pattern can provide unique information on the structure 
of reflection nebulae and their relationship to the illuminating sources. Specifically, polarimetric images can be used to identify deeply embedded sources (DESs), even though they cannot be detected directly in the near-infrared (e.g., Weintraub \& Kastner 1993; Yao et al. 2000), and to disentangle the geometry and structure of outflow cavities (Yamashita et al. 1987; Minchin et al. 1991a).

In this paper, we present for the first time the $J H K^{\prime}$ imaging polarimetry for S87 and $K$-band spectroscopy from 2.0 to $2.35 \mu \mathrm{m}$ for the infrared sources in it. Our polarimetry observations reveal a deeply embedded source associated with infrared nebulae, and allow us to investigate the properties of the illuminating source, nebular structure, and outflow activity in S87. The moderate-resolution spectrometry offers physical information about the young stellar objects (YSOs) in S87. We describe in Sect. 2 the observations and present the results in Sect. 3. The observational results are discussed in Sect. 4 and summarized in Sect. 5.

\section{Observations}

\subsection{Imaging polarimetry}

Observations were carried out on 1997 October 21 with the $1.88 \mathrm{~m}$ telescope at Okayama Astrophysical Observatory, using the infrared camera OASIS (Okayama Astrophysical System for Infrared Imaging and Spectroscopy; Okumura et al. 2000). OASIS, equipped with a NICMOS3 $\mathrm{HgCdTe}$ array, provides a field of view of $4.2 \times 4.2 \operatorname{arcmin}^{2}$ with a plate scale of 0.97 pixel $^{-1}$. A polarimeter, which consists of a rotating achromatic half-wave plate and a fixed cold polarizer, was attached to the OASIS. Such a configuration provides $\sim 4^{\prime}$ field of view. The instrumental polarization was measured to be less than $1 \%$.

S87 was observed in the $J, H$, and $K^{\prime}(2.16 \mu \mathrm{m})$ bands in step-and-integrate mode. Five dithered sets of images were obtained through each filter. Each set consists of four images taken consecutively at four positions of the wave plate stepped by 22.5 . At each position of the wave plate, integration time of 10,20 , and $30 \mathrm{~s}$ were taken for the $J, H$, and $K^{\prime}$ bands, respectively. The images were reduced using the standard IRAF routines with the same procedures as described in Yao et al. (1997). The Stokes parameter images were established from the reduced images at the four position angles. The zero position angle of the polarization images was calibrated through observations toward the polarized source AFGL $2591\left(\theta_{J}=171^{\circ}\right.$, $\theta_{H}=167^{\circ}, \theta_{K}=171^{\circ}$; Minchin et al. 1991b). The $F W H M$ of the seeing disk was measured to be $\sim 1^{\prime \prime} .8$. The $5 \sigma$ limiting magnitudes at $J, H$, and $K^{\prime}$ bands are 18.0, 16.5, and $15.2 \mathrm{mag} \mathrm{pixel}^{-1}$, respectively.

\subsection{K-band Spectroscopy}

The spectra of S87E and S87W were obtained with OASIS on 1999 November 18 and 2000 November 24, respectively. The spectral resolution of OASIS was set to be $\lambda / \Delta \lambda \sim 500$ with a $300 \mathrm{~mm}^{-1}$ grating and a 2 '. 4 wide slit. The wavelength coverage was from 2.0 to $2.35 \mu \mathrm{m}$. The slit was about $230^{\prime \prime}$ long and was aligned roughly along the east-west direction. In the observations, the slit was centered on the $K$-band peak of S87E and

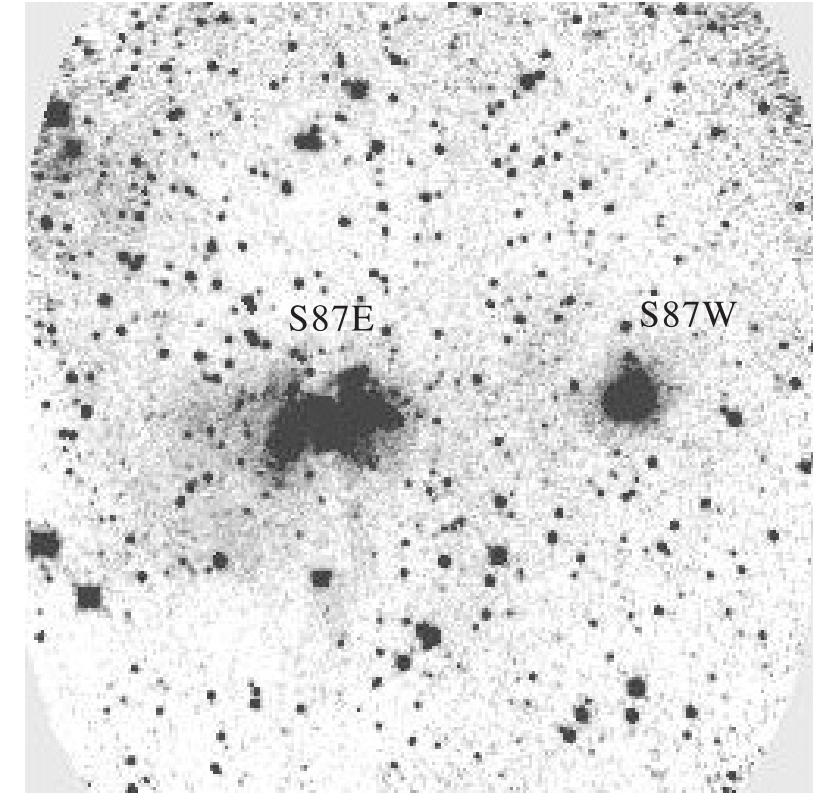

Fig. 1. $K^{\prime}$ image of S87 in an area of $4^{\prime} \times 4^{\prime}$. North is up and east to the left. The nebulae noted by Chen et al. (2003) are labelled.

S87W. The objects were observed 10 times with the telescope dithered along the slit to reduce the effects of bad pixels and cosmic rays. Each exposure time was $3 \mathrm{~min}$, and the telescope was guided during the exposure by monitoring nearby optically visible stars with the slit-viewer of the camera. Standard stars were also observed for atmospheric correction and flux calibration. The seeing condition during the observations was $\sim 1$ '. 8 .

Each spectrum frame was dark-subtracted and flat-fielded. The flat field was constructed by two sets of dome flat frames taken with an illuminating lamp on and off. The spectra were extracted using the IRAF APALL task. The extracted spectra were then level-adjusted to each other and median combined to produce a final spectrum. The spectrum was further divided by A0 V standard star (HR 9019) reduced in the same manner to remove atmospheric absorption features; $\mathrm{Br} \gamma$ absorption in the A0 V standard had been removed with the SPLOT task after being divided by the late-type standard (HR 9079), which shows little Bry absorption (Wallace \& Hinkle 1997). The spectrum was finally multiplied by a Planck function of an $9790 \mathrm{~K}$ blackbody, representative of the A0 V star. The accuracy of flux level was estimated to be $\sim 20 \%$ from the change of signals obtained at different slit positions.

\section{Results}

Figure 1 shows the $K^{\prime}$ image of S87, in which two bright nebulae presented in Paper I are labelled. S87E is an M-shaped nebula associated with a young cluster $\left(3 \sim 4 \times 10^{6} \mathrm{yr}\right.$; Paper I). The symmetry axis, passing through the nebula, is in the direction from northeast to southwest. Located $\sim 1.5$ arcmin to the west is the $\mathrm{S} 87 \mathrm{~W}$ nebula with a fairly round shape. $K^{\prime}$ brightness contour maps of the two nebulae are presented in Fig. 2. The offsets are in arcsec from the astrometry origin $\left(\operatorname{RA}(1950)=19^{\mathrm{h}} 44^{\mathrm{m}} 13^{\mathrm{s}} .1, \operatorname{Dec}(1950)=24^{\circ} 28^{\prime} 03^{\prime \prime}\right)$ and 


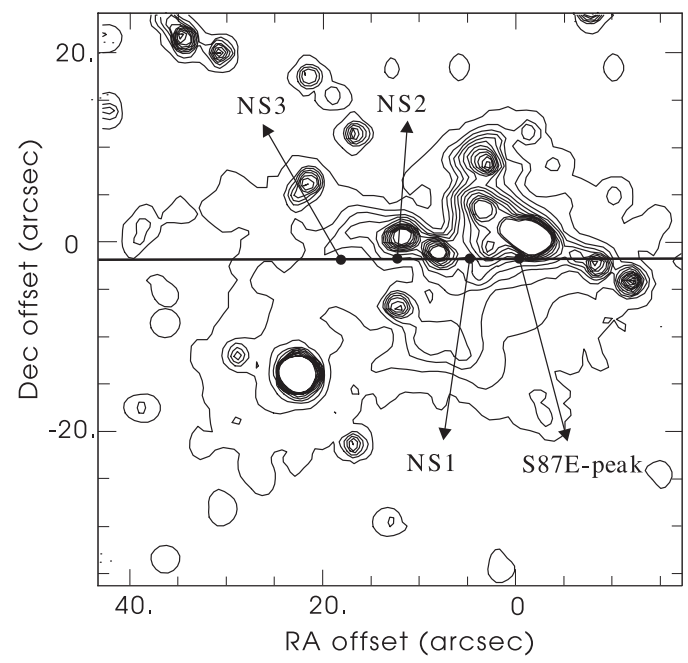

(a)

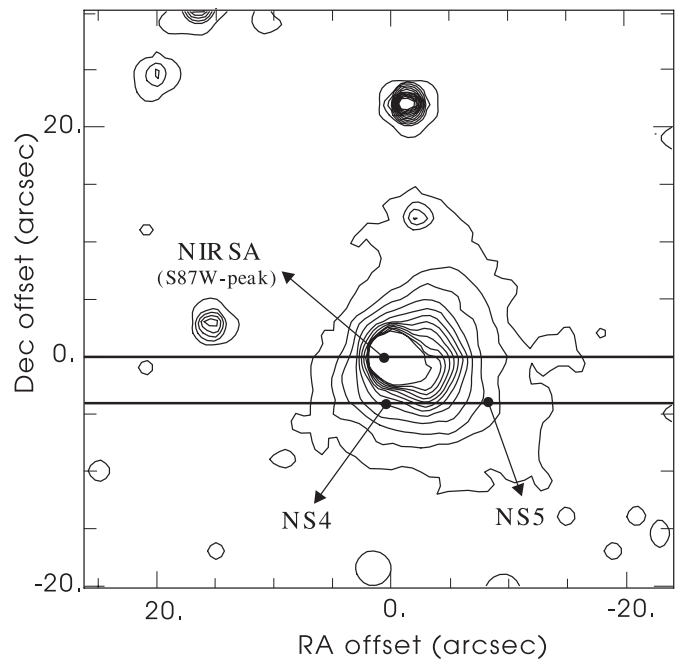

(b)

Fig. 2. a) $K^{\prime}$-band brightness contour map of S87E. The offsets are in arcsecs from the S87E-peak $\left[R A(1950)=19^{\mathrm{h}} 44^{\mathrm{m}} 13^{\mathrm{s}} \cdot 1\right.$, Dec(1950) $=$ $24^{\circ} 28^{\prime} 03^{\prime \prime}$ ], the $K^{\prime}$ peak of S87E. b) $K^{\prime}$-band brightness contour map of S87W. The contours are drawn from 11.6 to $15.2 \mathrm{mag}^{\mathrm{p}}$ pixel ${ }^{-1}$ with steps of 0.3 mag pixel ${ }^{-1}$. Axes offsets are in arcsecs from the $K^{\prime}$ peak of S87W [NIRS A; RA $(1950)=19^{\mathrm{h}} 44^{\mathrm{m}} 13^{\mathrm{s}} \cdot 1$, Dec $(1950)=24^{\circ} 28^{\prime} 03^{\prime \prime}$ ]. The "NS 1-5" in two images represent the positions of the sources in $K$-band spectrometry observations.

Table 1. Software aperture polarimetry of the reflection knots in S87E and S87W.

\begin{tabular}{cccccccccc}
\hline \hline Knot & $\begin{array}{c}\Delta x^{a} \\
(\operatorname{arcsec})\end{array}$ & $\begin{array}{c}\Delta y^{a} \\
(\operatorname{arcsec})\end{array}$ & $\begin{array}{c}\text { Aperture }^{b} \\
(\operatorname{arcsec})\end{array}$ & $\begin{array}{c}P_{J} \\
(\%)\end{array}$ & $\begin{array}{c}\theta_{J} \\
(\mathrm{deg})\end{array}$ & $\begin{array}{c}P_{H} \\
(\%)\end{array}$ & $\begin{array}{c}\theta_{H} \\
(\mathrm{deg})\end{array}$ & $\begin{array}{c}P_{K} \\
(\%)\end{array}$ & $\begin{array}{c}\theta_{K} \\
(\mathrm{deg})\end{array}$ \\
\hline DES-S87E & 6.0 & -2.1 & 5.0 & $7 \pm 0.25$ & 21 & $3 \pm 0.8$ & 11 & $2 \pm 0.7$ & 89 \\
A & 12.6 & -1.0 & 6.0 & $25 \pm 1.2$ & 19 & $10 \pm 1.6$ & 6 & $7 \pm 1.5$ & 5 \\
B & 1.5 & 4.5 & 6.0 & $22 \pm 2.5$ & 47 & $13 \pm 1.4$ & 44 & $11 \pm 2.3$ & 43 \\
C & 4.8 & -14.8 & 8.0 & $32 \pm 1.5$ & 107 & $20 \pm 4.5$ & 106 & $16 \pm 1.2$ & 91 \\
D & -78.5 & 5.8 & 6.0 & $31 \pm 1.5$ & 86 & $22 \pm 2.0$ & 97 & $12 \pm 1.2$ & 98 \\
E & -79.0 & -3.7 & 6.0 & $29 \pm 3.8$ & 88 & $19 \pm 2.4$ & 92 & $18 \pm 1.8$ & 79 \\
\hline
\end{tabular}

${ }^{a}$ The position are offset from $K$-peak of S87E.

${ }^{b}$ Software aperture employed for polarimetry.

$\left(\operatorname{RA}(1950)=19^{\mathrm{h}} 44^{\mathrm{m}} 07^{\mathrm{s}} .1, \operatorname{Dec}(1950)=24^{\circ} 28^{\prime} 07^{\prime \prime} .8\right)$, respectively, for which we assume the $K$ peak of S87E and S87W.

The polarization vector maps for $\mathrm{S} 87 \mathrm{E}$ at $J, H$, and $K^{\prime}$ are presented in Figs. 3a-3c, respectively. It should be noted that the signal-to-noise $(\mathrm{S} / \mathrm{N})$ at $K^{\prime}$ is relatively low compared to that at $J$ and $H$. A centrosymmetric pattern is exhibited in the S87E nebula at all three wavelengths, especially the in $H$ band. Referring to the procedure described by Weintraub \& Kastner (1993), we located a polarization centroid at $\alpha_{1}=$ $19^{\mathrm{h}} 44^{\mathrm{m}} 13.55, \delta_{1}=24^{\circ} 28^{\prime} 02^{\prime \prime} .5$ (B1950), with positional uncertainties of $\sim 1^{\prime \prime}$ in the north-south direction and $\sim 1^{\prime \prime}$.5 in the east-west direction. In the discussion below, this polarization centroid is demonstrated to be a deeply embedded source in S87E (Sect. 4.1.1). To the southeast and northwest of the polarization centroid, a pair of polarized nebular knots are found, which are labelled as "A" and "B" in Fig. 3b. Simultaneously, a moderate polarized elongation extending from the polarization centroid to the southwest is labelled as "C" in Fig. 3b. Table 1 gives the degree of polarization and position angle (PA) of polarization for the centroid and nebular knots "A-C" in the $J, H$ and $K^{\prime}$ bands. Directly to the west of the polarization centroid, the polarization vectors at $K^{\prime}$ deviate from a pure centrosymmetric pattern, and polarization degrees are particularly low, generally less than $10 \%$ (Fig. 3c). This disordered region includes the $K$ peak of S87E and some red sources (see Fig. 2a), which may interfere with the arrangement of $K^{\prime}$ polarimetric vectors in this region.

Figure 4 presents the $\left[J-K^{\prime}\right]$ color image of the S87E nebula. In the image, the nebula shows different colors in the eastern and western area. The reddest color is seen in the northern part of the S87E nebula, while the bluest color is seen in the southwest part of S87E with an elongation to the southwest, corresponding to the polarized elongation found on polarization maps. This elongation also coincides with the blueshifted lobe of $\mathrm{CO}$ outflow. If the bluer colors imply less extinction, this elongation could suggest a cavity structure.

Figures 5a-5c display polarization vector maps for S87W in $J, H$ and $K^{\prime}$, respectively. Offsets are in arcsec from NIRS A, which is saturated in the polarimetric images at all three wavelengths and is identifiable as a circular area of zero 


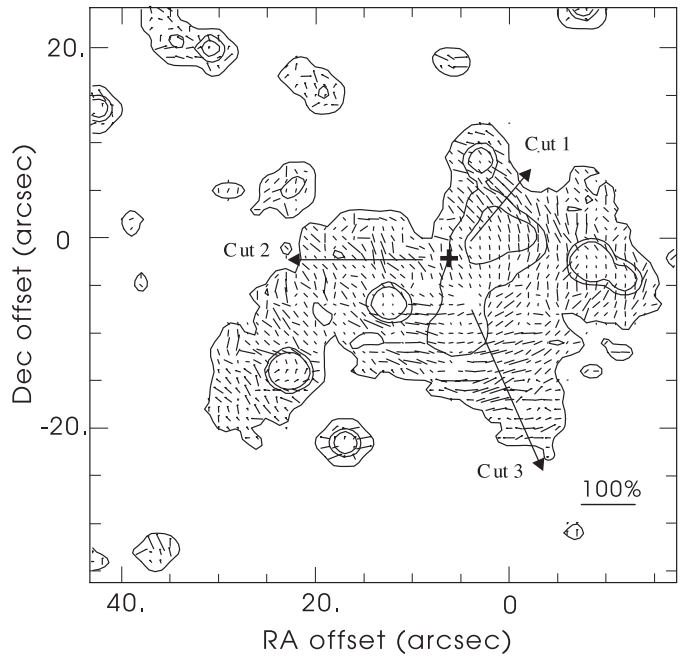

(a)

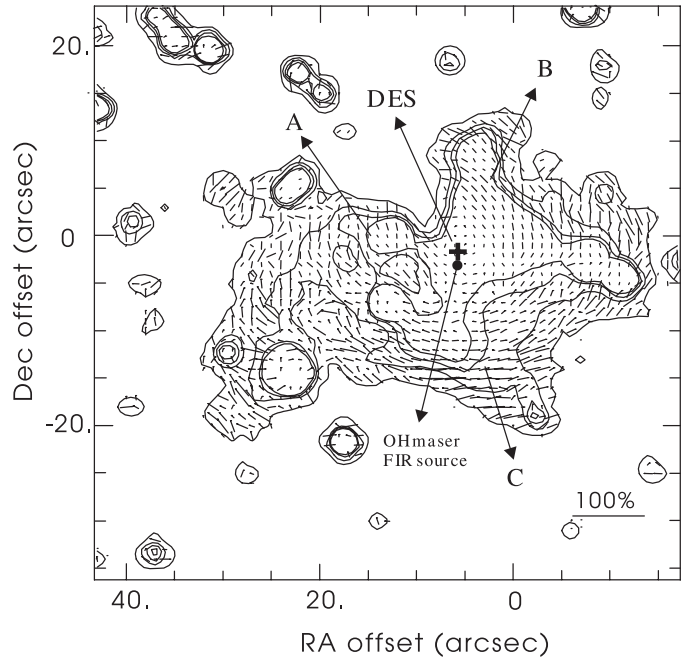

(b)

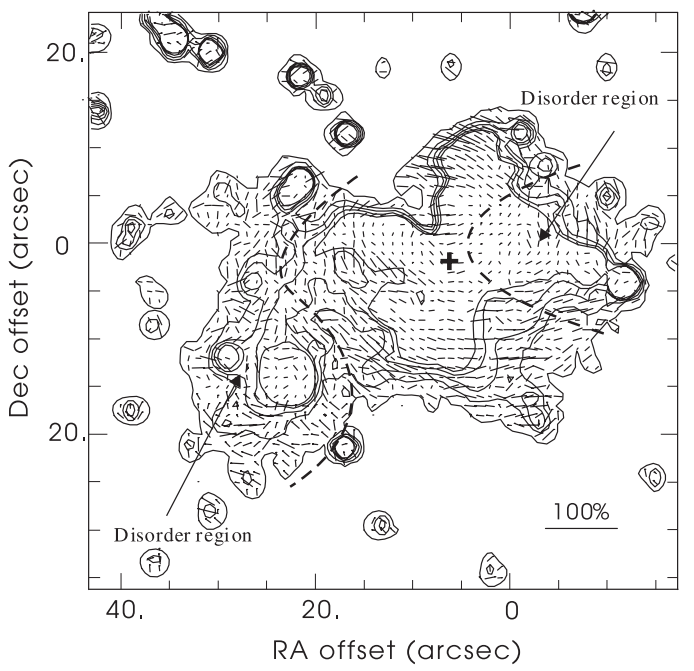

(c)

Fig. 3. a) $J$-band polarization map of S87E with the same axis offsets as Fig. 2a. The length of the vectors represents the percentage polarization, and the orientation of the vectors represents the position angle of polarization with respect to the equatorial coordinate system. The $100 \%$ polarization vector is shown at bottom right of the frame. The superposed contours are shown in linear scale to mark the relative position of vectors. b) The same as a, but at $H$. c) The same as a, but at $K^{\prime}$. The positions of the DES are marked by plus signs in three frames. The reflection knots are denoted as A-C in Fig. 3 b.

polarization. The polarization vectors in $J$ and $H$ bands roughly show a centrosymmetric pattern around NIRS A. In the $K^{\prime}$ band, for the low $\mathrm{S} / \mathrm{N}$, the alignment of polarization vector is a little irregular. Two highly polarized knots are found to the north and south of NIRS A, which are labelled as "D" and "E" in Fig. 5a. Both the polarization degree and position angle in $J$, $H, K^{\prime}$ for knots "D" and "E" are also listed in Table 1.

The spectra of S87E and S87W are shown in Figs. 6a and 6 b, respectively. In our observations, the $K$-band peaks of S87E and S87W were centered on the slits. We also searched for emission from nebula or nearby stars along the long slit of S87E. When such emission was found, the spectrum was extracted in the same manner as for the peak of S87E. The positions of these extracted sources are given in Table 2 and marked by "NS $1-5$ " in Fig. 2 . Some $K$-band spectral features of young stars can be found in the spectra, such as $\mathrm{Br} \gamma(2.166 \mu \mathrm{m}), \mathrm{H}_{2}$ $(2.122,2.223,2.248 \mu \mathrm{m})$ and $\mathrm{NaI}(2.206 \mu \mathrm{m})$. We regard the features as "detected" if they are more than $3 \sigma$ above the local continuum. Equivalent widths $(E W)$ were measured over a $0.01 \mu \mathrm{m}$ wide interval centered at the detected lines ( $1 \sigma$ of the equivalent widths is typically $\sim 0.5 \AA$; see Ishii et al. 2001). The equivalent widths and fluxes measured for the $\mathrm{Br} \gamma, \mathrm{H}_{2}$ $v=1-0 \mathrm{~S}(1)$, and $\mathrm{H}_{2} v=1-0 \mathrm{~S}(0)$ lines are also listed in Table 2 .

It can be found in Fig. 6 that the continuum of the "S87E-peak" is the reddest and rises towards longer wavelengths, and those of other objects are relatively flat or blue. Both $\mathrm{H}_{2}$ 1-0 S(1) and 1-0 S(0) emission lines are detected in all spectra. In the S87E nebula (Fig. 6a), the spectrum of the "S87E-peak" is the only example that does not show 
Table 2. Equivalent widths and fluxes of the observed objects.

\begin{tabular}{|c|c|c|c|c|c|c|c|c|}
\hline \multirow[b]{2}{*}{ Object } & \multicolumn{2}{|c|}{ Positions $^{a}$} & \multicolumn{2}{|c|}{$\operatorname{Br} \gamma$} & \multicolumn{2}{|c|}{$\mathrm{H}_{2} v=1-0 \mathrm{~S}(1)$} & \multicolumn{2}{|c|}{$\mathrm{H}_{2} v=2-1 \mathrm{~S}(1)$} \\
\hline & $\begin{array}{c}\Delta x \\
(\operatorname{arcsec})\end{array}$ & $\begin{array}{c}\Delta y \\
(\operatorname{arcsec})\end{array}$ & $\begin{array}{c}\text { Flux } \\
\left(10^{-17} \mathrm{~W} \mathrm{~m}^{-2}\right)\end{array}$ & $\begin{array}{c}E W^{b} \\
(\AA)\end{array}$ & $\begin{array}{c}\text { Flux } \\
\left(10^{-17} \mathrm{~W} \mathrm{~m}^{-2}\right)\end{array}$ & $\begin{array}{l}E W \\
(\AA)\end{array}$ & $\begin{array}{c}\text { Flux } \\
\left(10^{-17} \mathrm{~W} \mathrm{~m}^{-2}\right)\end{array}$ & $\begin{array}{l}E W \\
(\AA)\end{array}$ \\
\hline S87E-peak & 0.0 & 0.0 & $\ldots$ & $\ldots$ & $2.4 \pm 0.50$ & $10.5 \pm 1.1$ & ... & $\ldots$ \\
\hline NS 1 & 5.1 & 0.0 & $4.2 \pm 0.38$ & $55.4 \pm 4.0$ & $2.5 \pm 0.45$ & $30.8 \pm 2.9$ & $0.71 \pm 0.22$ & $10.2 \pm 2.4$ \\
\hline NS 2 & 12.8 & 0.0 & $3.2 \pm 0.23$ & $45.7 \pm 5.5$ & $1.6 \pm 0.35$ & $20.1 \pm 2.9$ & $0.41 \pm 0.14$ & $5.5 \pm 2.7$ \\
\hline NS 3 & 17.7 & 0.0 & $0.93 \pm 0.16$ & $35.1 \pm 4.5$ & $2.2 \pm 0.38$ & $80.1 \pm 6.5$ & $0.83 \pm 0.23$ & $32.6 \pm 4.1$ \\
\hline NIRS A & -79.5 & 4.8 & $5.1 \pm 0.44$ & $14.0 \pm 2.6$ & $1.5 \pm 0.16$ & $10.8 \pm 3.8$ & $\ldots$ & $\ldots$ \\
\hline NS 4 & -78.9 & 0.0 & $0.79 \pm 0.21$ & $10.6 \pm 2.6$ & $1.9 \pm 0.25$ & $32.6 \pm 4.6$ & $0.51 \pm 0.11$ & $10.1 \pm 1.6$ \\
\hline NS 5 & -87.2 & 0.0 & $\ldots$ & $\ldots$ & $3.2 \pm 0.23$ & $19.7 \pm 3.5$ & $0.55 \pm 0.26$ & $19.1 \pm 3.6$ \\
\hline
\end{tabular}

${ }^{a}$ The positions are offset from $K$-peak of S87E.

${ }^{b}$ A positive $E W$ corresponds to emission.

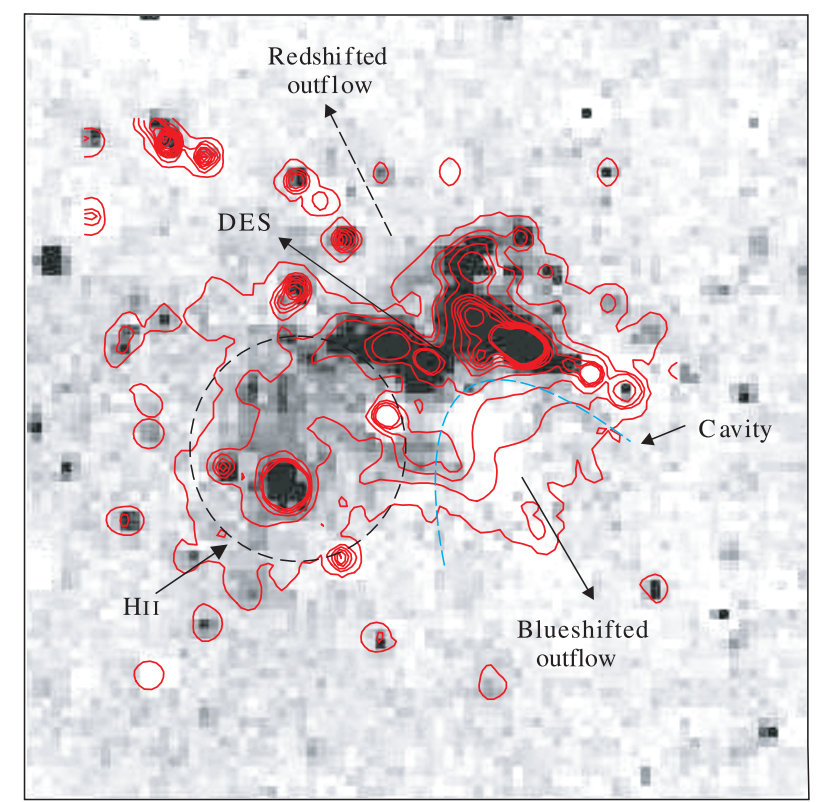

Fig. 4. $J-K^{\prime}$ color image of S87E. The $J-K^{\prime}$ color is linearly scaled from 0 mag (white) to 5 mag (black). The superposed contour map is same as the $K^{\prime}$-contour in Fig. 2a. Deeply embedded source is labelled "DES". The CO molecular outflow is marked by a solid arrow (blueshifted) and dashed arrow (redshited). A dashed circle delineates the HII region.

$\mathrm{Br} \gamma$ emission and instead shows relatively strong $\mathrm{H}_{2}$ emission. About 5". 1 east of "S87E-peak", strong $\mathrm{Br} \gamma$ emission $(E W=55.4 \pm 4.0 \AA), \mathrm{H}_{2}$ emissions $(E W=30.8 \pm 2.9 \AA$ for $1-0 \mathrm{~S}(1), 11.9 \pm 2.9$ for $1-0 \mathrm{~S}(0)$, and $10.2 \pm 2.4$ for $2-1 \mathrm{~S}(1)$ ), and CIV emission $(E W=14.2 \pm 2.1 \AA)$ are found in the spectrum of "NS 1". A spectrum of a star located $\sim 12$ ". 8 east was also taken, which shows $\operatorname{Br} \gamma(E W=45.7 \pm 5.5 \AA)$ and $\mathrm{H}_{2}$ emis$\operatorname{sion}(E W=20.1 \pm 2.9 \AA$ for $1-0 \mathrm{~S}(1), 11.8 \pm 3.0$ for $1-0 \mathrm{~S}(0)$, and $5.5 \pm 2.7$ for $2-1 \mathrm{~S}(1))$. Going farther east, relatively weak $\mathrm{Br} \gamma$ and $\mathrm{H}_{2}$ emissions are found (NS 3). In the S87W nebula (Fig. 6b), strong Br $\gamma$ emission $(E W=14.0 \pm 2.6 \AA)$, $\mathrm{H}_{2}$ emissions $(E W=10.8 \pm 3.8 \AA$ for $1-0 \mathrm{~S}(1))$, and Na I emission $(E W=5.5 \pm 1.5 \AA)$ are detected in the spectrum of the "S87W-peak".

\section{Discussion}

\subsection{The nebular complex of S87E}

\subsubsection{The deeply embedded source}

Through the orientation of polarimetry vectors, one polarization centroid is inferred in the polarimetric maps of S87E. However, no point sources in the near-infrared images were found to coincide with this polarization centroid. Analogous to many observations (e.g., Weintraub \& Kastner 1993, 1996; Yao et al. 2000), the centroid implies a deeply embedded source in the nebula of S87E (hereafter DES-S87). A low degree of polarization is observed toward this source (see Table 1). Small but rather centrosymmetric polarimetric patterns are found to the northwest, southeast and southwest of DES-S87 (Fig. 3).

Several observations, referred to earlier, strongly support the existence of DES-S87. The first evidence is the $\mathrm{OH}$ maser spot detected in S87E $\left(\alpha_{1}=19^{\mathrm{h}} 44^{\mathrm{m}} 13^{\prime \prime} .5, \delta_{1}=24^{\circ} 28^{\prime} 00^{\prime \prime}\right.$, B1950), which coincides well with the position of DES-S87 (Braz \& Epchtein 1983; Braz \& Sivagnanam 1987; Wouterloot et al. 1993). Since there is no other infrared source closer to the maser spot, we suggest that the $\mathrm{OH}$ maser is powered by the DES-S87. The second evidence is the $1.3 \mathrm{~mm}$ dust continuum emission at the position of DES-S87 (Chini et al. 1986), which generally comes from a dust envelope around the YSOs. Thirdly, the far-infrared source IRAS 19442+2428 $\left(19^{\mathrm{h}} 44^{\mathrm{m}} 13.5,24^{\circ} 28^{\prime} 00^{\prime \prime}\right.$, B 1950$)$ detected at this position provided another piece of evidence. Additionally, the spectral feature of $3.1 \mu \mathrm{m} \mathrm{H}_{2} \mathrm{O}$ ice absorption, which is ubiquitously found in spectra of YSOs deeply embedded in molecular clouds, was detected in S87E in the $3 \mu \mathrm{m}$ spectra survey of YSOs by Ishii et al. (1998). Although the aperture size of their observations was too large ( 5 arcsec or 9 arcsec) to decide an accurate observed position, the $3.1 \mu \mathrm{m} \mathrm{H}_{2} \mathrm{O}$ ice feature indicates the presence of deeply embedded sources in the S87E nebulae, which is consistent with our polarimetry result. 


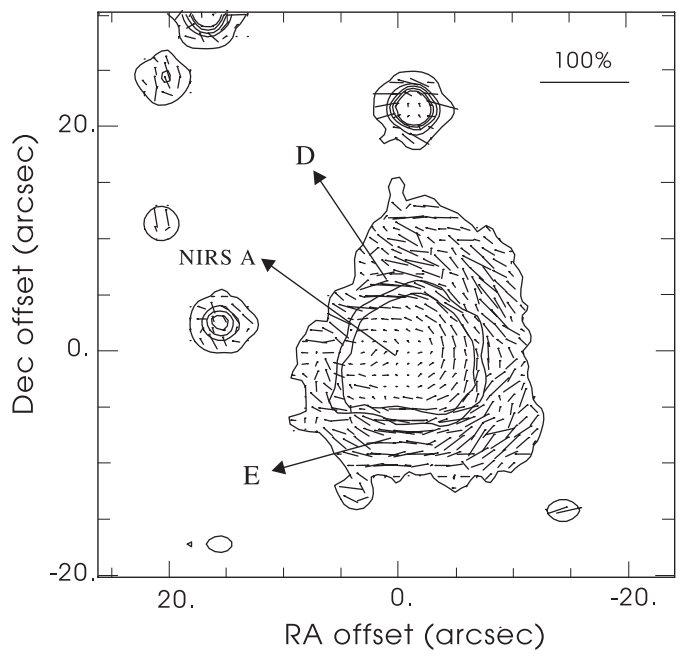

(a)

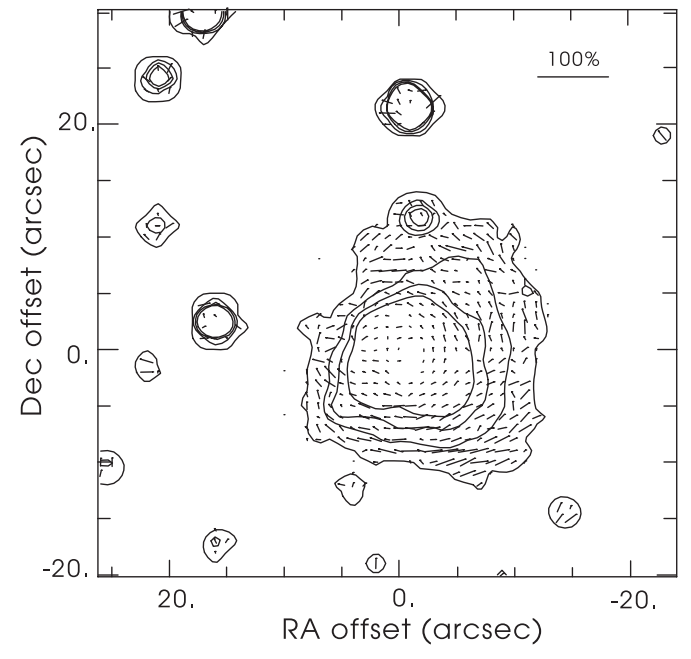

(b)

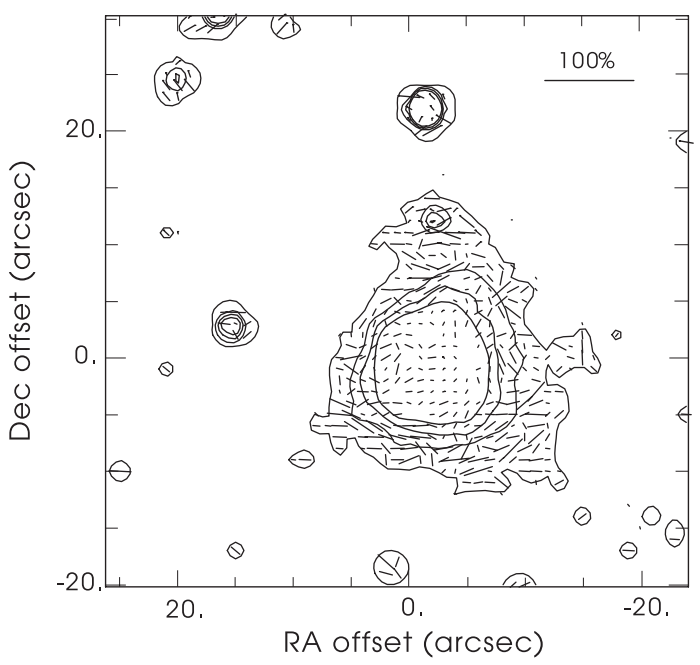

(c)

Fig. 5. a) $J$-band polarization map of $\mathrm{S} 87 \mathrm{~W}$ with the same axis offsets as Fig. $2 \mathrm{~b}$. The length of the vectors represents the percentage polarization, and the orientation of the vectors represents the position angle of polarization with respect to the equatorial coordinate system; The $100 \%$ polarization vector is shown at top right of the frame. The superposed contours are shown in linear scale to mark the relative position of vectors. b) The same as a, but at $H$. c) The same as a, but at $K^{\prime}$. The reflection knots are denoted as "D" and "E" in Fig. 5a.

To investigate the wavelength dependence of polarization in the reflection nebulosities around DES-S87, we calculated the ratios of $H$ to $J$ and $K^{\prime}$ to $H$ percentage polarization $\left(P_{H} / P_{J}\right.$ and $P_{K^{\prime}} / P_{H}$, respectively) along radial cuts starting from DES-S87 as described by Minchin et al. (1991a). The cuts begin at a radial distance 3 arcsec from DES-S87 to avoid contamination. The ratios for each pixel along the cuts were averaged. The results are given in Table 3 . The average values of both $P_{H} / P_{J}$ and $P_{K^{\prime}} / P_{H}$ along cut $1(0.82 \pm 0.10$ and $0.77 \pm 0.14$ respectively $)$, cut $2(0.70 \pm 0.11$ and $0.84 \pm 0.14$ respectively), and cut $3(0.83 \pm 0.11$ and $0.72 \pm 0.16$ respectively $)$ are clearly less than unity. Thus there is a general trend for the observed polarization around DES-S87 to decrease with wavelength, which indicates single Rayleigh scattering in the reflection nebulosities. At the same time, this decreasing percent polarization as a function of wavelength provides further evidence that DES-S87 is a thermal source. If the DES-S87 was a
Table 3. The wavelength dependence of scattered radiation from DES.

\begin{tabular}{cccc}
\hline \hline Cut $^{a}$ & $n^{b}$ & $P_{H} / P_{J}$ & $P_{K} / P_{H}$ \\
\hline 1 & 11 & $0.82 \pm 0.10$ & $0.77 \pm 0.14$ \\
2 & 15 & $0.70 \pm 0.11$ & $0.84 \pm 0.12$ \\
3 & 21 & $0.83 \pm 0.11$ & $0.72 \pm 0.16$ \\
\hline
\end{tabular}

${ }^{a}$ Three cuts were taken for DES-S87E: cut 1 is to the northwest of the source; cut 2 is to the east of the source; cut 3 is along the blueshifted outflow axis.

${ }^{b}$ The number of pixels covered by each cut.

purely scattering center, then the degree of polarization should increase or remain constant with increasing near-infrared wavelengths regardless of grain size distribution (Pendleton et al. 1990), which is contrary to what we observe. 


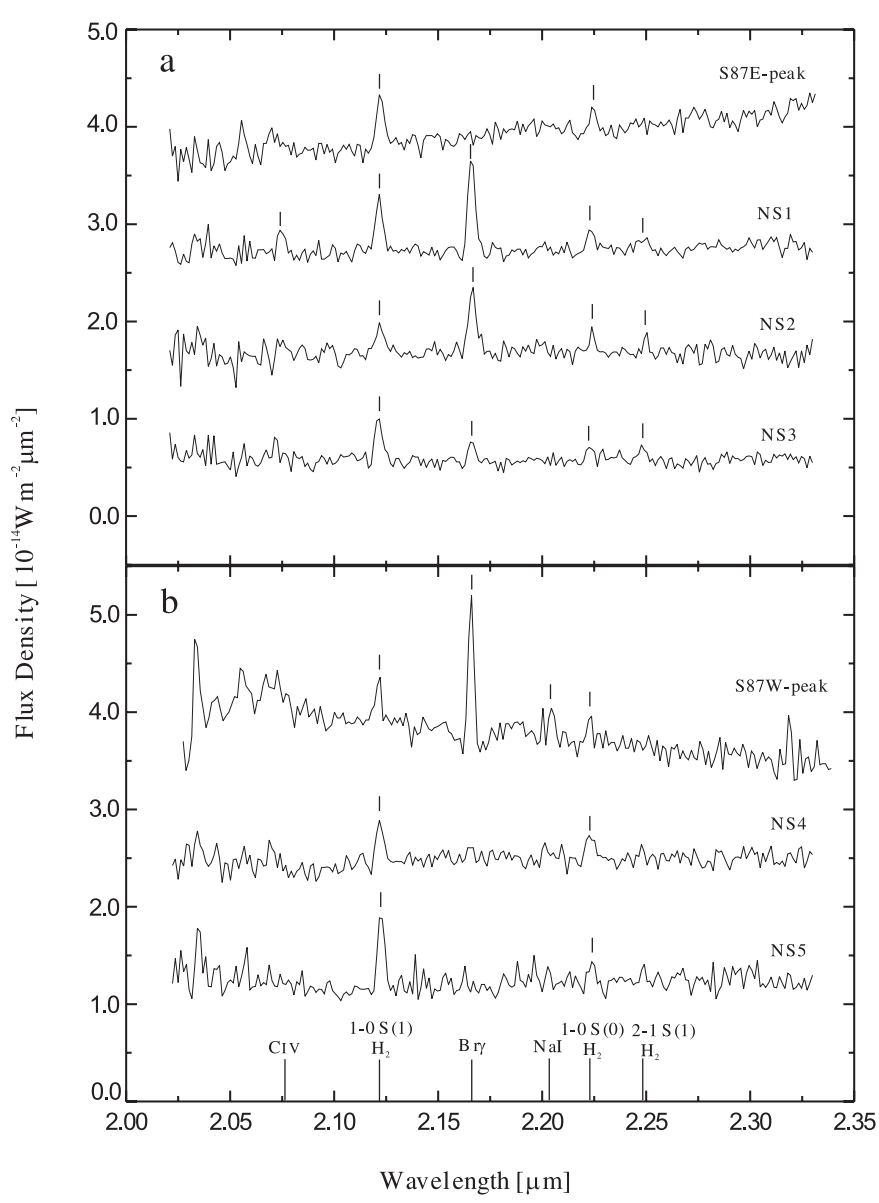

Fig. 6. $K$-band moderate resolution $(\lambda / \Delta \lambda \sim 500)$ spectra of the observed sources in S87Ea) and S87W b). Fluxes are shifted vertically for clarity. All line emissions identified are indicated.

Yao et al. (2000) have proposed that DESs could be a manifestation in the near-infrared of the earliest phase of massive star formation and possess characteristics similar to hot molecular cores. The lines of evidence above indicate that DES-S87 is an extremely young massive star, probably in the earliest stage of protostellar evolution of massive stars. In light of these facts, we suggest that it is the youngest source in S87. The technique of using a polarization centroid to locate an embedded protostar has successfully been employed by Weintraub \& Kastner (1993, 1996) and Yao et al. (2000). However, the DESs unveiled by near-infrared polarimetric imaging need further confirmation with longer wavelength or high-resolution (sub)millimeter-wave observations. The DESs revealed by Weintraub et al. in near-infrared polarimetric studies of molecular outflow regions, such as L1287 (IRAS 00338+6312; Weintraub \& Kastner 1993), LkH $\alpha 234$ (PS1; Weintraub et al. 1994), and AFGL 437 (WK 34; Weintraub \& Kastner 1996), all have been confirmed by the follow-up $L^{\prime}$-band $(3.8 \mu \mathrm{m})$ imaging (Weintraub et al. 1996). There are also increasing reports of confirmations based on (sub)millimeter-wave observations, such as IRAS 20126+4104 (Hofner et al. 1999) and S233B (Yao et al. 2000; Jiang et al. 2001; Beuther et al. 2002). We believe that high resolution observations at (sub)millimeter-wave will be helpful to further understand the properties of the DES-S87.

\subsubsection{Bipolar CO outflow in S87E}

The bipolar outflow centered on S87E cluster is a massive outflow, whose blueshifted and redshifted $\mathrm{CO}$ flows run roughly in the southwest and northeast directions, respectively. A closeup view of the outflow by Barsony (1989) suggested a massive ( $\approx 20 M_{\odot}$ ) pre-main-sequence driving source, which is still embedded in its parent molecular cloud. Nevertheless, the identification of a driving source for the outflow is not straightforward in the near-infrared images, since some bright infrared sources are located in the center of cluster and the resolution of $\mathrm{CO}$ observations are not high enough to point out the driving source.

Weintraub \& Kastner $(1993,1996)$ have argued that the DESs are most likely the drivers of molecular outflows, which could be consistent with the more recent concept that the youngest protostars tend to drive the most energetic and most highly collimated outflows (e.g., Richer et al. 2000). As discussed above, DES-S87 is proposed as the youngest source in S87 powering $\mathrm{OH}$ maser and $1.3 \mathrm{~mm}$ dust continuum emission. At the same time, the strong $\mathrm{Br} \gamma$ and $\mathrm{H}_{2}$ emissions found in the $K$-band spectrum of DES-S87 implies it powers strong wind or outflow. The mass of DES-S87 is estimated to be $\sim 20 M_{\odot}$ based the $K$-band spectrum (see Sect. 4.3), which is in close agreement with the mass suggested by Barsony (1989). According to its young and massive nature, we suggest that DES-S87 is the driving source of bipolar CO outflow in S87.

The $\left[J-K^{\prime}\right]$ color image in Fig. 4 exhibits an elongated cavity structure to the southwest of DES-S87. This cavity is spatially coincident with the blueshifted lobe of the $\mathrm{CO}$ outflow, which could indicate that the cavity is excavated by the blue lobe of outflow approaching the observer and sweeping away the material in the molecular cloud. Along the axis of the cavity to the southwest, the polarization degrees increase with the distance from DES-S87 (Fig. 3). Yamashita et al. (1987) and Minchin et al. (1991b) had calculated the radial dependence of polarization for parabolic outflows with varying inclination of the outflow axis. Comparing our data to their models, the radial dependence of polarization degrees in the cavity could be fitted to the slab model of outflows at an inclination of $\sim 60^{\circ}$ out of the plane of the sky (Minchin et al. 1991b, Fig. 10). It is approximately consistent with the inclination angle proposed by Barsony $\left(\approx 50^{\circ} ; 1989\right)$.

\subsubsection{The structure of reflection nebulae: A quadrupolar outflow?}

The nebulae of S87E show complex morphology in the polarization images. This morphology could be roughly divided into three areas by polarimetry vectors: the first is the centrosymmetric pattern predominating over most of the northern part of the nebulae (hereafter referred to as the northern nebula); the second is the bright nebula in the southwest (hereafter the southwest nebula); the third is the disordered polarimetry area in the southeast that coincides with the HII region in S87 
(hereafter the ionized nebula). This feature is consistent with the $\left[J-K^{\prime}\right]$ color of S87E (Fig. 4) that the reddest color is seen around the northern nebula, the bluest color is seen in the southwest nebula, and an intermediate color is seen in the ionized nebula. The polarimetric vectors in the ionized nebula is arranged irregularly, especially at $K^{\prime}$ (Fig. 3c). In our near-infrared images, this region is relatively weak (Figs. 1 and $2 \mathrm{a})$. The degree of polarization in the region is in the range of $10 \% \sim 20 \%$. The polarization vectors in this region have large errors because the unpolarized intensity of the nebula is low. We suggest that the most polarimetric vectors in this region are spurious given the weakness of nebula.

The overall morphology of the northern nebula shows a bipolar structure. The whole nebula is centered on DES-S87 and extends roughly in the southeast-northwest direction. This structure is confirmed by the $\left[J-K^{\prime}\right]$ image of S87E, in which the northern nebula also presents a bipolar structure with DES-S87 on the apex. Two polarized nebular knots, "A" and "B" (Figs. 3 and 4), have nearly the same projected distance from DES-S87 and display good alignment with DES-S87. As previously noted, strong Br $\gamma$ emission and $\mathrm{H}_{2}$ emissions are detected at the position of DES-S87, which suggests strong stellar wind and outflow activity. Furthermore, $\mathrm{H}_{2}$ emission may imply the presence of a circumstellar envelope around DES-S87 (see below). All the factors fit the general picture of star formation involving a central source, a flattened disk/envelope, and a bipolar outflow. The two polarized nebular knots ("A" and "B") thus most likely indicate a pair of reflection lobes of bipolar outflow from DES-S87. The large degrees of polarization $(20 \sim 30 \%)$ in the lobes, as well as the similar infrared colors seen to the east and west of DES-S87, further indicate that the axis of the bipolar lobes is not far from the plane of the sky.

The southwest nebula traces the cavity evacuated by the blue lobe of bipolar CO outflow. Suggested by the bipolar structure of the $\mathrm{CO}$ outflow, it is possible that the southwest nebula is also one reflection lobe of the bipolar nebula. However, the other lobe has not been detected in our images. A scenario for the production of this feature is that the other lobe traces the redshifted lobe of $\mathrm{CO}$ outflow that is moving away from us and obscured by dense cloud material of the northern nebula. Thus, there are maybe two bipolar nebulae detected around DES-S87: one lies nearly in the plane of the sky with a pair of lobes projecting in the northwest-southeast direction; the other extends in the northeast-southwest direction with a pair of lobes tracing the redshifted and blueshifted $\mathrm{CO}$ outflow, respectively. This nebular structure suggests that DES-S87 may drive two distinct bipolar outflows nearly perpendicular to each other. The configuration of ${ }^{13} \mathrm{CO}$ outflows in the $\mathrm{S} 87$ could be support for the quadrupolar outflow hypothesis. In Fig. 12 of Barsony (1989), red and blue ${ }^{13} \mathrm{CO}$ wings expand to the northeast and southwest, respectively; furthermore, both red and blue line centers extend in the southeastnorthwest direction. This configuration is in close agreement with the quadrupolar structure suggested by our polarimetric images.

Such quadrupolar (multipolar) outflow structures have been observed in a few cases in millimeter-wavelength observations (e.g., IRAS 20050+2720; Bachiller et al. 1995) and near-infrared $\mathrm{H}_{2}$ observations (e.g., Hodapp \& Ladd 1995; Ladd \& Hodapp 1997). Yao et al. (1998) reported a quadrupolar outflow in S140 based on polarimetry imaging observations. It can be found that these quadrupolar (multipolar) outflow structures are closely associated with extremely young and deeply embedded sources. However, the physics behind these multipolar outflows remains unknown. Two plausible explanations have been proposed: a single time- and angle-variable driving source has generated the multipolar outflows, or multiple unresolved driving sources are independently responsible for each bipolar outflow (e.g., Anglada et al. 1996; Ladd \& Hoddap 1997). In S87, there is currently no observational evidence to exclude either possibility. In preliminary discussions, we tend to adopt the latter explanation for S87, because: (1) there are some red sources in the center of bipolar CO outflow, thus we cannot exclude the possibility that other sources, not DES-S87, drive the bipolar $\mathrm{CO}$ outflow; (2) within the positional errors of the polarimetric centroid $\left(\sim 1^{\prime \prime} .5\right)$, there are possibly multiple embedded sources which are unresolved by our polarimetric vectors. As suggested previously, further high resolution observations at longer wavelengths will be helpful to clarify the properties of $\mathrm{S} 87$.

\subsection{The nebular complex of S87W}

The well-defined centrosymmetric pattern with high degrees of polarization $(30 \sim 35 \%)$ found in the S87W nebula indicates single Rayleigh scattering off small dust grain in the nebula (Fischer et al. 1996; Lucas \& Roche 1998). The polarization vectors in a $\sim 10^{\prime \prime}$ region around the illuminating source NIRS A form an elliptical pattern (Figs. 5a, 5b). Small degrees of polarization are seen along the southeast-northwest direction. Such a polarization pattern, commonly referred to as a "polarization disk", has been widely observed in reflection nebulae associated with young stellar objects, such as AFGL 2591 (Tamura et al. 1991; Minchin et al. 1991b), HL Tau (Weintraub et al. 1995) and Mon R2 (Yao et al. 1997). According to the dust scattering models of Bastien \& Ménard (1988), Whitney \& Hartmann (1993) and Fischer et al. (1994, 1996), a polarization disk can be expected for scattered light emerging from an optically thick disk around the illuminating source. The elongated direction of the polarization disk indicated the orientation of the presumed disk plane. The fluorescent $2.206 \mu \mathrm{m}$ $\mathrm{NaI}$ emission line found in the spectrum of NIRS A provides further evidence for the presence of a circumstellar disk, where the $\mathrm{NaI}$ region can be shielded from the direct stellar radiation since sodium has a low first ionization potential $(5.1 \mathrm{eV})$ (McGregor et al. 1988).

The morphology of the S87W nebula is roughly round, compared to the complex morphology of S87E. It is generally recognized that nebulae evolve as the YSOs in them do, while the relationship between the evolution of nebulae and YSOs still remains unclear. Jiang et al. (2001) classified the structure of the nebulae into three groups: (1) compact, referring to those nebulae with small and roughly round geometry; (2) bipolar, referring to those showing bipolar geometry 
and centrosymmetric polarization patterns; (3) complex, referring to those of irregular geometry but being disentangled by polarization patterns, and proposed that while YSOs evolved, the associated nebulae evolved morphologically from complex to bipolar and then to compact. According to their definition, the structures of nebulae S87E and S87W are "complex" and "compact", respectively. Since the YSOs in S87W are relatively more evolved than those in S87E (Paper I), our polarimetric results confirm the conjecture of Jiang et al. (2001). Furthermore, the polarimetric imaging of the S87E nebula is also consistent with the statistical result found in Table 2 of Jiang et al. (2001) that in the cluster regions, when the YSOs are DESs, the associated nebulae all display complex structure.

\subsection{K-band spectra of S87}

\subsubsection{Circumstellar environment of S87}

The $K$-band spectra of S87E and S87W are presented in Figs. $6 \mathrm{a}$ and $6 \mathrm{~b}$, respectively. The $\mathrm{Br} \gamma$ and $\mathrm{H}_{2}$ emission lines found in our spectra are frequently seen in the near-infrared spectra of YSOs. The $2.166 \mu \mathrm{m} \mathrm{Br} \gamma$ line is the only hydrogen line expected in this wavelength region. This line emission is generally thought to occur in stellar winds close to the stars (Ishii et al. 2001 and references therein). In the $K$-band spectroscopy study of YSOs, $\mathrm{Br} \gamma$ emission is correlated with the source luminosities rather than the spectral energy distributions (SEDs) (Persson et al. 1984; Greene \& Lada 1996; Ishii et al. 2001). It indicates that the emission is closely associated with the mass of YSOs. On the contrary, $\mathrm{H}_{2}$ emission clearly depends on the SEDs from class I to class II (Greene \& Lada 1996; Ishii et al. 2001). It is rarely detected in class III SEDs, but mostly detected in class I SEDs. The fact that $\mathrm{H}_{2}$ emission is found for those with class I SEDs indicates that the emission is closely associated with the circumstellar envelopes.

It has been known that collisional excitation in shocked gas is the dominant $\mathrm{H}_{2}$ emission mechanism in protostellar flows. However, it should be kept in mind that UV fluorescence is also an important mechanism of $\mathrm{H}_{2}$ excitation. In general, the line ratio of $\mathrm{H}_{2}$ 2-1 $\mathrm{S}(1) / 1-0 \mathrm{~S}(1)$ lines is calculated to infer the emission mechanism such that $\sim 0.1$ are observed in shocked regions and $\sim 0.5$ in the photodissociation regions (PDRs) (Shull \& Bechwith 1982; Martini et al. 1999). The small ratios of $2-1 \mathrm{~S}(1) / 1-0 \mathrm{~S}(1)$ found in our spectra could support excitation by shocks. However, there is another possibility, that the $\mathrm{H}_{2}$ emission occurs in dense PDRs with densities of $\geq 10^{5} \mathrm{~cm}^{-3}$ where the $2-1 \mathrm{~S}(1) / 1-0 \mathrm{~S}(1)$ ratios could be similar to those in shocked regions (Draine \& Bertoldi 1996; Luman et al. 1998). To differentiate between excitation in shocks and in dense PDRs, the lines ratios of 2-1 S(1)/1-0 S(1) and $1-0 \mathrm{~S}(0) / 1-0 \mathrm{~S}(1)$ are shown in Fig. 7 compared with several theoretical models. It is noted that although the wavelengths of these transitions are similar, the high extinction toward S87 regions $\left(A_{\mathrm{v}} \sim 15\right.$; Paper I) can significantly alter the observed line ratios from their intrinsic values. For modification, each line ratio would be multiplied by a factor of $\sim 0.8$, causing the positions in Fig. 7 to move toward the origin. In Fig. 7, our spectral data are away from the region of "low

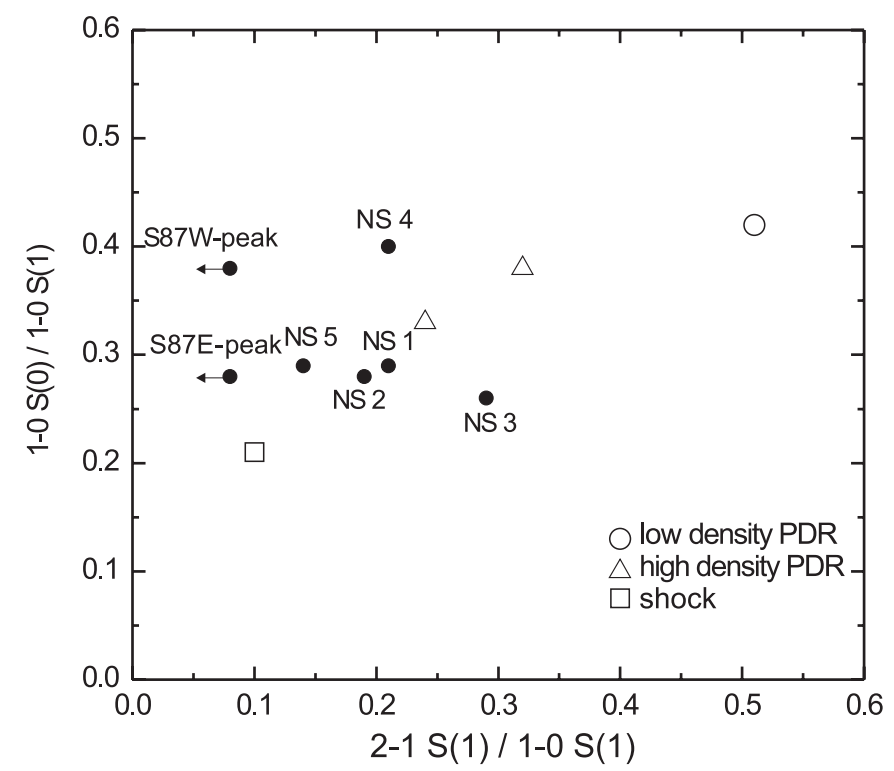

Fig. 7. 2-1 $S(1) / 1-0 \quad S(1)$ vs. 1-0 $S(0) / 1-0 \quad S(1)$ diagram for the $\mathrm{H}_{2}$ emissions. The data (filled circles) are compared to the theoretical predictions (open circle) of Draine \& Bertoldi (1996) for pure fluorescence in a low density PDR $\left(n_{\mathrm{H}}=10^{4} \mathrm{~cm}^{-3}, \chi=10^{2}\right.$, $T_{0}=500 \mathrm{~K}$ ), emission from a PDR model (triangle) at higher density $\left(n_{\mathrm{H}}=10^{6} \mathrm{~cm}^{-3}, \chi=10^{4}, T_{0}=500 \mathrm{~K}\right.$ and $n_{\mathrm{H}}=10^{6} \mathrm{~cm}^{-3}$, $\chi=10^{5}, T_{0}=1000 \mathrm{~K}$ ) that matched data for the Orion Bar (Luhman et al. 1998), and shock excitation (square) indicative of a shock $\left(T_{0}=\right.$ 2000 K; Blank \& van Dishoeck 1987).

density PDR" ( $\left.n_{\mathrm{H}}=10^{4} \mathrm{~cm}^{-3}, \chi=10^{2}, T_{0}=500 \mathrm{~K}\right)$ and mainly distributed in the zone between the "shock" $\left(T_{0}=\right.$ $2000 \mathrm{~K})$ and "high density PDR" $\left(n_{\mathrm{H}}=10^{6} \mathrm{~cm}^{-3}, \chi=10^{5}\right.$, $\left.T_{0}=1000 \mathrm{~K}\right)$. Considering the small line ratio of $2-1 \mathrm{~S}(1) / 1-0$ $\mathrm{S}(1)$ in our spectra, we suggest that the $\mathrm{H}_{2}$ emissions in $\mathrm{S} 87$ are generated by the cooperative action of shocks and dense PDR. It possibly implies that $\mathrm{H}_{2}$ emission in $\mathrm{S} 87$ originates in the inner high-density regions of the shocked stellar wind. In short, the $\mathrm{Br} \gamma$ and $\mathrm{H}_{2}$ emission lines in our $K$-band spectra show properties of strong stellar wind, envelope/disk, shocked gas, and dense PDRs in the circumstellar environment of S87E and S87W.

No absorption feature is detected in our spectra. The absence of near-infrared absorption features is likely consistent with continuum veiling of photospheric features by the large near-infrared excess (Paper I). This excess is probably caused by warm circumstellar material and may indicate active starforming activity. Higher resolution with high signal-to-noise spectroscopy at $J$ or $H$ will be helpful to further understand the properties of these objects.

\subsubsection{K-band spectrum of deeply embedded source}

It can be found in Fig. 2a that the extracted position of "NS 1" coincides well with the position of DES-S87. Because our spectra were extracted from $\sim 4$ " aperture along 2.'4 wide slit, we consider that the spectrum of "NS 1" covers most emission from DES-S87. Thus, the spectrum of "NS 1 " in fact represents the $K$-band spectrum of DES-S87, 
in which strong $\mathrm{Br} \gamma$ emission $(E W=55.4 \pm 4.0 \AA), \mathrm{H}_{2}$ emission $(E W=30.8 \pm 2.9 \AA$ for $1-0 \mathrm{~S}(1), 11.9 \pm 2.9$ for $1-0$ $\mathrm{S}(0)$, and $10.2 \pm 2.4$ for $2-1 \mathrm{~S}(1))$, and $2.078 \mu \mathrm{m}$ CIV emission $(E W=14.2 \pm 2.1 \AA)$ are found (Fig. 6a and Table 2). It is possibly the first $K$-band spectroscopy observation of a deeply embedded source.

The $2.078 \mu \mathrm{m}$ CIV line found in the spectrum is usually apparent in mid-O and late-O type stars (Hanson et al. 1996). Both CIV and Bry in emission without NIII and HeI lines may imply kO7-kO8 spectral type of YSOs (cf. Table 6 of Hanson et al. 1996). (The " $\mathrm{k}$ " in this notation is an indication that these are $K$-band spectral types, not optical MK spectral types.). Spectral classes of $\mathrm{kO} 7-\mathrm{kO} 8$ correspond closely to $\mathrm{MK}$ spectral classes of O8-O9 (Hanson et al. 1996). This range of spectral types (O8-O9) corresponds to effective temperatures of $36500 \sim 34500 \mathrm{~K}$ and a luminosity of $4 \sim 6 \times 10^{4} L_{\odot}$ assuming a ZAMS star (cf. Table 1 of Panagia 1973). On the other hand, it has been shown that there is a correlation between the source luminosity and the luminosity of $\mathrm{Br} \gamma$ line (Persson et al. 1984; Greene \& Lada 1996; Ishii et al. 2001). The Bry flux of DES-S87 corresponds to a luminosity of $\sim 10^{4} L_{\odot}$ (cf., Fig. 2 in Ishii et al. 2001). If an extinction of $A v=15$ is taken into account (Paper I), the luminosity would be corrected to $\sim 4 \times 10^{4} L_{\odot}$. Both luminosities derived from the CIV line and $\operatorname{Br} \gamma$ line are a little larger than the bolometric luminosity of $3.5 \times 10^{4} L_{\odot}$ determined from energy distribution from 0.001 to $100 \mathrm{~mm}$ of S87 (Barsony 1989). Barsony (1989) suggested the B0 ZAMS luminosity be an underestimate of the true luminosity of an embedded source. In this paper we suggest O8 ZAMS luminosity to be an upper limit of the true luminosity of the DES-S87. Therefore, DES-S87 could be reconciled as a deeply embedded pre-main-sequence source with a mass of $20 M_{\odot}$. This mass is equal to the mass suggested by Barsony (1989) based on radio observations.

\section{Conclusions}

Near-infrared polarimetric imaging has been carried out for the massive star-forming region $\mathrm{S} 87$, and moderate-resolution spectrometry from 2.0 to $2.35 \mu \mathrm{m}$ has been performed for the infrared sources in the region. The main results can be summarized as follows:

1. The polarimetric patterns of S87E reveal a deeply embedded source, DES-S87, associated with reflection nebulae. With exciting $\mathrm{OH}$ maser and $1.3 \mathrm{~mm}$ dust continuum emission, DES-S87 is proposed to be the youngest star in the region.

2. We suggest that DES-S87 is the driving source of bipolar $\mathrm{CO}$ outflow in the region. A blue cavity is revealed by $\left[J-K^{\prime}\right]$ color image to the southwest of DES-S87. The radial dependence of polarization degrees in the cavity could be fitted by the slab model of outflow at an inclination of $\sim 60^{\circ}$ out of the plane of the sky.

3. The nebular complex of S87E can be divided into three areas. The northern nebula, exhibiting a bipolar structure, may represent a bipolar outflow in the northwest-southeast direction. The Southwest nebula traces the blueshifted
$\mathrm{CO}$ outflow and corresponds to the cavity structure in the $\left[J-K^{\prime}\right]$ color image. The structure of the nebular complex may represent a quadrupolar outflow driven by DES-S87.

4. A well-defined centrosymmetric pattern is found in the nebula S87W with high degrees of polarization, which indicates single Rayleigh scattering off small dust grains in the reflection nebula. The polarimetric vectors around NIRS A show a polarization disk feature, consistent with our spectroscopy observations.

5. Strong $\mathrm{Br} \gamma$ emission and $\mathrm{H}_{2}$ emissions are found in the $K$-band spectra, indicating the presence of envelope, stellar wind, shocked gas, and dense PDRs in the circumstellar environment of S87E and S87W. The $K$-band spectrum of DES-S87 is also obtained, which indicates the high mass $\left(20 M_{\odot}\right)$ and youth characteristics.

Acknowledgements. The authors wish to thank the OAO staff for their assistance in the observations. X. P. Chen thanks Dr. Y. F. Chen for his kind help in the manuscript revision. This work is supported by NSFC grants 10133020 and 10273022, and Ministry of Science and Technology G19990754.

\section{References}

Anglada, G., Rodriguez, L. F., \& Torrelles, J. M. 1996, ApJ, 473, L123 Bachiller, R., Fuente, A., \& Tafalla, M. 1995, ApJ, 445, L51

Bally, J. 1981, Ph.D. Thesis, University of Massachusetts at Amherst Bally, J., \& Predmore, R. 1983, ApJ, 265, 778

Bally, J., \& Lada, C. J. 1983, ApJ, 265, 824

Barsony, M. 1989, ApJ, 345, 268

Bastien, P., \& Ménard, F. 1988, ApJ, 326, 334

Beuther, H., Schilke, P., Gueth, F., et al. 2002, A\&A, 387, 931

Blair, G. N., Dinger, A. S. C., \& Dickinson, D. F. 1980, AJ, 85, 161

Blank, J. H., \& van Dishoeck, E. F. 1987, ApJ, 322, 412

Braz, M. A., \& Epchtein, N. 1983, A\&AS, 54, 167

Braz, M. A., \& Sivagnanam, P. 1987, A\&A, 181, 19

Cesaroni, R., Felli, M., \& Jenness, T. et al. 1999, A\&A, 345, 949

Chen, H., Tafalla, M., Greene, T. P., Myers, P. C., \& Wilner, D. J. 1997, ApJ, 475, 163

Chen, Y. F. 2001, Ph.D. Thesis, Purple Mountain Observatory

Chen, Y. X., Zheng, X. W., Yao, Y. Q., Yang, J., \& Sato, S. 2003, A\&A, 401, 185

Chini, R., Kreysa, E., Mezger, P. G., \& Gemünd, H.-P. 1986, A\&A, 54, L8

Clemens, D. P. 1985, ApJ, 295, 422

Drain, B. T., \& Bertoldi, F. 1996, ApJ, 468, 269

Fischer, O., Henning, Th., \& Yorke, H. W. 1994, A\&A, 284, 187

Fischer, O., Henning, Th., \& Yorke, H. W. 1996, A\&A, 308, 863

Greene, T. P., \& Lada, C. J. 1996, AJ, 112, 2184

Henkel, C., Haschick, A. D., \& Güsten, R. 1986, A\&A, 165, 197

Hodapp, K.-W., \& Ladd, E. F. 1995, ApJ, 453, 715

Hofner, P., Cesaroni, R., Rodriguez, L. F., \& Marti, J. 1999, A\&A, 345L, 43

Ishii, M., Nagata T., Sato, S., et al. 1998, AJ, 116, 868

Ishii, M., Nagata T., Sato, S., et al. 2001, AJ, 121, 3191

Jiang, Z. B., Yao, Y. Q., Yang, J., et al. 2001, AJ, 122, 313

Kurtz, S., Churchwell, E., \& Wood, D. O. S. 1994, ApJS, 91, 659

Kurtz, S., Watson, A. M., Hofner, P., Otte, B. 1999, ApJ, 514, 232

Ladd, E. F., \& Hodapp, K.-W. 1997, ApJ, 474, 749

Lucas, P. W., \& Roche, R. F. 1998, MNRAS, 299, 699

Luhman, K. L., Engelebracht, C. W., \& Luhman, M. L. 1998, ApJ, 499, 799 
Martini, P., Sellgren, K., \& DePoy D. L., 1999, ApJ, 526, 772

McGregor, P. J., Hyland, A. R., \& Hillier, D. J. 1988, ApJ, 324, 1071

Minchin, N. R., Hough, J. H., McCall, A., et al. 1991b, MNRAS, 249, 707

Minchin, N. R., Hough, J. H., McCall, A., et al. 1991b, MNRAS, 251, 508

Okumura, S., Nishihara, E., Watanabe, E., et al. 2000, PASJ, 52, 931

Pendleton, Y. J., Tielens, A. G. G. M., \& Werner, M. W. 1990, ApJ, 349, 107

Richer, J. S., Shepherd, D. S., Cabrit, S., Bachiller, R., \& Churchwell, E. 2000, in Protostars and Planets IV, ed. V. Mannings, A. P. Boss, \& S. S. Russell (Tucson: Univ. Arizona Press), 867

Sharpless, S. 1959, ApJS, 4, 257

Shull, J. M., \& Bechwith, S. 1982, ARA\&A, 20, 163

Scoville, N., Kleinmann, S. G., Hall, D. N. B., \& Ridgway, S. T. 1983, ApJ, 275, 201

Tamura, M., Gatley, I., Joyce, R. R., et al. 1991, ApJ, 378, 611
Wallace, L., \& Hinkle, K. 1997, ApJS, 111, 445

Weintraub, D. A. \& Kastner, J. H. 1993, ApJ, 411, 767

Weintraub, D. A. \& Kastner, J. H. 1996, ApJ, 458, 670

Weintraub, D. A., Kastner, J. H., \& Mahesh, A. 1994, ApJ, 420, L87

Weintraub, D. A. \& Kastner, J. H., \& Whitney, B. A. 1995, ApJ, 452, L141

Weintraub, D. A., Kastner, J. H., Gatley, I., \& Merrill, K. M. 1996, ApJ, 468L, 45

Whitney, B. A., \& Hartmann, L. 1993, ApJ, 402, 605

Wouterloot, J. G. A., Brand, J., \& Fiegle, K. 1993, A\&AS, 98, 589

Yamashita, T., Sato, S., Nagata, T., et al. 1987, A\&A, 177, 258

Yao, Y. Q., Ishii, M., Nagata, T., et al. 1997, ApJ, 490, 281

Yao, Y. Q., Ishii, M., Nagata, T., et al. 1998, ApJ, 500, 320

Yao, Y. Q., Ishii, M., Nagata, T., Nakaya, T., \& Sato, S. 2000, ApJ, 542,392 